\title{
Fibrinogen-like-protein 1 promotes the invasion and metastasis of gastric cancer and is associated with poor prognosis
}

\author{
YANG ZHANG ${ }^{1}$, HUI-XIA QIAO ${ }^{2}$, YONG-TAO ZHOU $^{3}$, LIANG HONG ${ }^{4}$ and JU-HUI CHEN ${ }^{3}$ \\ ${ }^{1}$ Department of Gastroenterology, Zhengzhou Central Hospital Affiliated to Zhengzhou University, Zhengzhou, \\ Henan 450007; ${ }^{2}$ Department of Spleen and Stomach, Xi'an Traditional Chinese Medicine Hospital, \\ Xi'an, Shanxi 710021; Departments of ${ }^{3}$ Abdominal Radiotherapy and ${ }^{4}$ Pathology, \\ Fujian Province Cancer Hospital, Fuzhou, Fujian 350014, P.R. China
}

Received January 19, 2018; Accepted May 22, 2018

DOI: $10.3892 / \mathrm{mmr} .2018 .9097$

\begin{abstract}
The protective role of fibrinogen-like-protein 1 (FGL1) in liver injury has been reported previously. However, there are few studies on FGL1 expression in gastric cancer (GC) tissues, and the role of FGL1 in GC remains unclear. The aim of the present study was to investigate the correlation between FGL1 expression and prognosis in GC patients. Data was downloaded from The Cancer Genome Atlas database, and 50 pairs of GC tissues and the corresponding non-tumor tissues were collected between 2008 to 2011. Furthermore, FGL1 expression was silenced in order to explore its role in SGC-7901 cell proliferation, invasion and migration using Cell Counting Kit-8, wound healing, Transwell invasion and migration assays, respectively. Finally, whether FGL1 is involved in epithelial-mesenchymal transition (EMT) regulation in SGC-7901 cells was determined by western blotting. The results revealed that FGL1 expression was upregulated in GC tissues, and the overall survival time of GC patients with high FGL1 expression levels was markedly shorter than that of GC patients with low FGL1 expression levels $(\mathrm{P}=0.005)$. In addition, silencing FGL1 significantly inhibited SGC-7901 cell proliferation, invasion and migration in vitro. Finally, western blot analyses indicated that knockdown of FGL1 markedly increased E-cadherin expression levels $(\mathrm{P}<0.01)$, and significantly decreased $\mathrm{N}$-cadherin $(\mathrm{P}<0.01)$ and vimentin expression levels $(\mathrm{P}<0.01)$, thereby suggesting that FGL1 may promote EMT. These results indicated that FGL1 has the potential to be a predictor in GC patients as well as a target for the treatment of GC.
\end{abstract}

Correspondence to: Dr Ju-Hui Chen, Department of Abdominal Radiotherapy, Fujian Province Cancer Hospital, 420 Fuma Road, Fuzhou, Fujian 350014, P.R. China

E-mail: chenjuhui6688@163.com

Key words: fibrinogen-like-protein 1, gastric cancer, prognosis, invasion and migration, epithelial to mesenchymal transitions

\section{Introduction}

Great progress has been made in the study of cancer including the mechanism of cancer, drug discovery and therapeutic modes, during the past 10 years (1). However, gastric cancer (GC) is one of the most common malignancies $(2,3)$, and the 5-year survival rate of GC patients remains very low (4). Since GC patients are often diagnosed at an advanced stage and because malignant proliferation, as well as lymphatic metastasis, frequently occurs in GC, few patients can be completely cured by surgery (5). Hence, the early diagnosis and treatment of gastric cancer are very necessary. Currently, biomarkers and regular clinicopathological parameters that can predict prognosis are limited; hence, few effective treatment options are available (6).

Fibrinogen-like-protein 1 (FGL1, also called FREP1 or hepassocin), a member of the fibrinogen family, is expressed mainly in the liver and was first cloned from human hepatocellular carcinoma $(7,8)$. Previous studies have demonstrated that the expression of FGL1 was increased in the regenerated liver and could stimulate $3 \mathrm{H}$-thymidine uptake in primary hepatocytes which implied that FGL1 facilitate hepatocyte proliferation (9). Moreover, recombinant FGL1 protected against liver injury in rats with fulminant hepatic failure (10). These observations suggest that FGL1 plays a role in liver regeneration and liver protection. In addition to its expression in the liver, FGL1 expression has also been reported in adipose tissue (8). Until now, no research has investigated FGL1 expression in GC tissues, and the role of FGL1 in GC remains large unclear.

In this study, we investigated the correlation between FGL1 expression and prognosis in GC patients. Besides, the effect of FGL1 on GC SGC-7901 cell proliferation, invasion and migration were also explored by silencing FGL1. Furthermore, the involvement of FGL1 in EMT regulation in SGC-7901 cells was determined by analyzing the expression of EMT markers using western blot. Our results indicated that high FGL1 expression was correlated with poor prognosis of GC patients and silencing FGL1 could inhibit SGC-7901 cell proliferation, invasion and migration.

\section{Materials and methods}

Data collection. The RNA-Seq gene expression data and clinical information of 375 gastric adenocarcinoma samples 
and 32 nomal gastric samples were downloaded from The Cancer Genome Atlas (TCGA; tcga-data.nci. Nih.gov/tcga). The difference of FGL1 expression between normal gastric tissue and gastric adenocarcinoma tissue was analyzed by using Student's t-test. The data collection process complies with all laws and regulations.

Patients and tissue samples. The GC cancer tissues and the corresponding adjacent tissues used in this study were collected from 50 patients who experienced a surgery for GC at Zhengzhou Central Hospital Affiliated to Zhengzhou University from 2008 to 2011. All patients did not undergo radiotherapy or chemotherapy before surgery. Histological diagnosis of gastric cancer is based on the World Health Organization (WHO) standards. Clinicopathological factors of the 7th edition of the (Union for International Cancer Control) UICC classification for gastric cancer were used to explore the importance of FGL1. Detailed clinicopathological information is shown in Table I. The present study was approved by the Ethics Committee of Zhengzhou Central Hospital Affiliated to Zhengzhou University (Henan, China). Written informed consent was obtained from all patients.

Cell culture. The Human Gastric cancer cell lines BGC-823, SGC-7901 and human gastric normal epithelial mucosa cell line GES-1 were all purchased from the Chinese Academy of Sciences and cultured in RPMI-1640 medium (Hyclone, USA) added with $10 \%$ serum, $100 \mathrm{U} / \mathrm{ml}$ penicillin, and $0.1 \mathrm{mg} / \mathrm{ml}$ streptomycin at $37^{\circ} \mathrm{C}$ with $5 \% \mathrm{CO}_{2}$. When cells growing into the logarithmic growth phase, single cell suspensions were prepared using trypsin (Solarbio, Beijing, China) and inoculated into 6-well plate for the subsequent tests.

Reverse transcription-quantitative polymerase chain reaction ( $R T$ - $q P C R)$. Total RNA was extracted using Ultrapure RNA kit (CwBio, Beijing, China) following the manufacturer's description. HiFiScript cDNA Synthesis kit (CwBio, Beijing, China) was used to perform RT-PCR to synthesize cDNA. Then the cDNA was used as template to perform qPCR. GAPDH was used as an internal reference gene. The following primer sequences were used: FGL1 forward, 5'-GCAAGGAGTCTG CTTCTGCT-3' and reverse, 5'-TGCCATGTTCCCCCTTGA AA-3'; and GAPDH forward 5'-GGAGCGAGATCCCTC CAAAAT-3', reverse 5'-GGCTGTTGTCATACTTCTCAT GG-3' (Genewiz, Beijing, China). qPCR was performed using SYBR Premix Ex Taq II kit (Takara Biotechnology Co., Ltd., Dalian, China) and carried out in a ABI 7500 system (Applied Biosystems, Carlsbad, CA, USA). qPCR procedure was as follows: $95^{\circ} \mathrm{C}$ for $5 \mathrm{~min}$, followed by 40 cycles of $5 \mathrm{sec}$ at $95^{\circ} \mathrm{C}$, $34 \mathrm{sec}$ at $60^{\circ} \mathrm{C}$, and then $72^{\circ} \mathrm{C}$ for $30 \mathrm{~min}$. All experiments were performed in triplicate and repeated for independently 3 times. The relative expression level of TGFBI was calculated using the $2-\Delta \Delta \mathrm{Cq}$ method (11).

Transfection. Transfection was performed using Lipofectamine 2000 (Invitrogen; Thermo Fisher Scientific, Inc., Waltham, MA, USA). When the cells in the 6-well plate grew to logarithmic period, the media was replaced with fresh complete culture medium $2 \mathrm{~h}$ before transfection. Cells were transfected with either FGL1 siRNA (siRNA1: 5'-AGGUGU
Table I. Clinicopathological characteristics of 50 gastric cancer patients.

\begin{tabular}{|c|c|}
\hline Variable & Case, n $(\%$ \\
\hline \multicolumn{2}{|c|}{ Age at diagnosis, years } \\
\hline$<60$ & $18(36)$ \\
\hline$\geq 60$ & $32(64)$ \\
\hline \multicolumn{2}{|l|}{ Sex } \\
\hline Female & $19(38)$ \\
\hline Male & $31(62)$ \\
\hline \multicolumn{2}{|l|}{ Grade } \\
\hline G1 & $3(6)$ \\
\hline G2 & $16(32)$ \\
\hline G3 & $31(62)$ \\
\hline \multicolumn{2}{|l|}{ Stage } \\
\hline I & $7(14)$ \\
\hline II & $15(30)$ \\
\hline III & $18(36)$ \\
\hline IV & $10(20)$ \\
\hline \multicolumn{2}{|c|}{ Pathologic-T } \\
\hline $\mathrm{T} 1$ & $4(8)$ \\
\hline $\mathrm{T} 2$ & $10(20)$ \\
\hline T3 & $17(34)$ \\
\hline $\mathrm{T} 4$ & $19(38)$ \\
\hline \multicolumn{2}{|c|}{ Pathologic-N } \\
\hline NO & $20(40)$ \\
\hline N1 & $4(8)$ \\
\hline $\mathrm{N} 2$ & $7(14)$ \\
\hline N3 & $19(38)$ \\
\hline \multicolumn{2}{|c|}{ Pathologic-M } \\
\hline M0 & $40(80)$ \\
\hline M1 & $10(20)$ \\
\hline
\end{tabular}

UCAGUUUCAUCCU-3' or SiRNA2: 5'-ACAGCAACAGGU CAAGAUC-3') or scramble siRNA (5'-CUAGAACUGGAC AACGACA-3') following manufacturer's procedure. After incubating the transfected cells in the incubator for $6 \mathrm{~h}$, the medium was replaced with complete medium. Forty eight $h$ later, the inhibitory efficiency of these siRNAs was verified by RT-qPCR and western blot assay, and the most effective siRNA were used for the following functional experiments.

Cell proliferation assay. After being transfected for $48 \mathrm{~h}$, cell suspensions were prepared and inoculated into 96-well plate with 1,000 cells for each well followed by culturing in a carbon dioxide incubator. The viability of cells were tested every $24 \mathrm{~h}$ by adding $10 \mu \mathrm{lCCK} 8$ chemicals (Solarbio), incubating at $37^{\circ} \mathrm{C}$ for $1.5 \mathrm{~h}$ and detecting the optical density (OD) at $450 \mathrm{~nm}$. The proliferation curves were drawn using Graph Pad Prism 5.

Colony formation assay. Single cell suspensions were prepared after transfected for $48 \mathrm{~h}$. And cells were plated in a $60 \mathrm{~mm}$ dish (500 cells/dish) containing $5 \mathrm{ml}$ culture medium. The dish was maintained at $37^{\circ} \mathrm{C}$ with $5 \% \mathrm{CO}_{2}$ and saturated humidity 
for 14 days. The experimental group was supplemented with $1 \mathrm{ml}$ of the supernatant liquid every 2-3 days and the control group with the same amount of PBS. The cultivation will be terminated when macroscopic colonies appeared in the dish. After removing the supernatant, the colonies were carefully washed by PBS. Then cells were fixed with $4 \%$ paraformaldehyde for 30 min followed by staining with $0.1 \%$ crystal violet for $30 \mathrm{~min}$. Next the dyes were washed using the running water and the number of clones was counted directly. All tests were performed in triplicate.

Wound healing assay. We performed wound healing assay to investigate the effect of FGL1 on SGC-7901 cell migration. Cell suspensions were prepared and seeded into 6-well plate with about $5 \times 10^{5}$ cells/well. After cultured for $24 \mathrm{~h}$, a $200 \mathrm{ml}$ micropipette tip was used to make the wounds. The widths of the wounds were photographed after 0 and $24 \mathrm{~h}$ scratching using an optical microscope (Olympus Corporation, Tokyo, Japan). The relative wounds of SGC-7901 cells transfected with FGL1 siRNA or scramble siRNA at $24 \mathrm{~h}$ were compared to the accordingly wounds at $0 \mathrm{~h}$.

Transwell invasion and migration assay. To further determine the role of FGL1 in cell invasion and migration, Transwell assays were performed. Transwell invasion chambers coated with Matrigel (100 $\mu \mathrm{l}$, diluted with serum-free medium at a ratio of 1:6) (BD Biosciences, Franklin Lakes, NJ, USA) was used to perform the invasion assays. Cell suspensions were prepared using serum-free medium after transfected for $24 \mathrm{~h}$ and transferred to the up chamber $\left(1 \times 10^{5}\right.$ cells/well). Then $500 \mu \mathrm{l}$ of complete medium were added to the lower chamber. After incubating at $37^{\circ} \mathrm{C}$ for $24 \mathrm{~h}$, the chamber was taken out, and the invaded cells were stained by $0.1 \%$ crystal violet stain. Then the number of the invaded cells was counted under a light microscope. Transwell migration assay was similar with Transwell invasion assay, but Matrigel was not needed and 5,000 cells were added to each well.

Western blot. After transfected for $48 \mathrm{~h}$, total protein were extracted using RIPA lysate supplemented with protease inhibitor. The proteins were heated at $95^{\circ} \mathrm{C}$ for $5 \mathrm{~min}$ and separated by $10 \%$ SDS-PAGE. Then proteins on the gel were electric transferred to a PVDF membrane (Millipore, Bedford, MA) and blocked in $5 \%$ skimmed milk for $1 \mathrm{~h}$. Subsequently, the membrane was incubated with the primary anti-body at $4^{\circ} \mathrm{C}$ overnight followed by washing with TBST for $3 \times 5 \mathrm{~min}$. Then the membrane was incubated with the secondary anti-body for $1 \mathrm{~h}$ and washed with TBST for $3 \times 5 \mathrm{~min}$. Enhanced chemiluminescence (ECL) plus detection kit (Thermo Fisher Scientific, Inc.) was used to detect signals of the protein. Quantity One software was used to perform the densitometric analysis of the bands. GAPDH was used as an intrinsic quality control. The ratio of the target protein to the internal reference protein is considered as the relative expression level.

Statistical analysis. The correlation between FGL1 expression and clinicopathological features were assessed using Chi-square $\left(\chi^{2}\right)$ test. The survival curve was drawn using Kaplan-Meier method and the difference between groups was assessed by log-rank test. The median of FGL1 expression
Table II. Correlation between FGL1 expression and clinicopathological features of $50 \mathrm{GC}$ patients.

\begin{tabular}{|c|c|c|c|}
\hline \multirow[b]{2}{*}{ Characteristics } & \multicolumn{2}{|c|}{ Expression of FGL1 } & \multirow[b]{2}{*}{ P-value } \\
\hline & Low (n) & High (n) & \\
\hline Age, years & & & 0.556 \\
\hline$<60$ & 8 & 10 & \\
\hline$\geq 60$ & 17 & 15 & \\
\hline Sex & & & 0.382 \\
\hline Female & 11 & 8 & \\
\hline Male & 14 & 17 & \\
\hline Grade & & & $0.041^{\mathrm{a}}$ \\
\hline $\mathrm{G} 1+\mathrm{G} 2$ & 13 & 6 & \\
\hline G3 & 12 & 19 & \\
\hline Pathologic-Stage & & & $0.023^{\mathrm{a}}$ \\
\hline $\mathrm{I}+\mathrm{II}$ & 15 & 7 & \\
\hline III+IV & 10 & 18 & \\
\hline Pathologic-T & & & 0.107 \\
\hline $\mathrm{T} 1+\mathrm{T} 2$ & 9 & 4 & \\
\hline $\mathrm{T} 3+\mathrm{T} 4$ & 16 & 21 & \\
\hline Pathologic-N & & & $0.021^{\mathrm{a}}$ \\
\hline No & 14 & 6 & \\
\hline $\mathrm{N} 1+\mathrm{N} 2+\mathrm{N} 3$ & 11 & 19 & \\
\hline Pathologic-M & & & 0.157 \\
\hline M0 & 22 & 18 & \\
\hline M1 & 3 & 7 & \\
\hline
\end{tabular}

${ }^{\mathrm{a}} \mathrm{P}<0$ 005. FGL1, fibrinogen-like-protein 1; GC, gastric cancer.

was used to divide the samples into high and low expression groups. The prognostic role of FGL1 in gastric adenocarcinoma was assessed using univariate and multivariate Cox proportional hazards analysis. SPSS 15.0 software (SPSS, Inc., Chicago, IL, USA) was used to perform the statistical analysis. The mean of multiple samples was compared using one-way analysis of variance analysis followed by Dunnett's post hoc test. $\mathrm{P}<0.05$ was considered to indicate a statistically significant difference.

\section{Results}

FGL1 was upregulated in gastric cancer tissues and associated with poor prognosis. Firstly, the expression of FGL1 was analyzed based on the data downloaded from TCGA database. As shown in Fig. 1A, the expression of FGL1 was significantly increased in gastric cancer tissues compared with the normal tissues $(\mathrm{P}<0.001)$. Then the expression of FGL1 of 50 patients in the gastric cancer tissues and the corresponding adjacent tissues were analyzed by qPCR and western blotting. We found that the expression of FGL1 in GC tissues were obviously higher than that in the adjacent tissues at both mRNA and protein levels $(\mathrm{P}<0.001$, Fig. $1 \mathrm{~B}$ and $\mathrm{C})$.

In order to further investigate the role of FGL1 in GC, the correlation of FGL1 expression with clinicopathological 

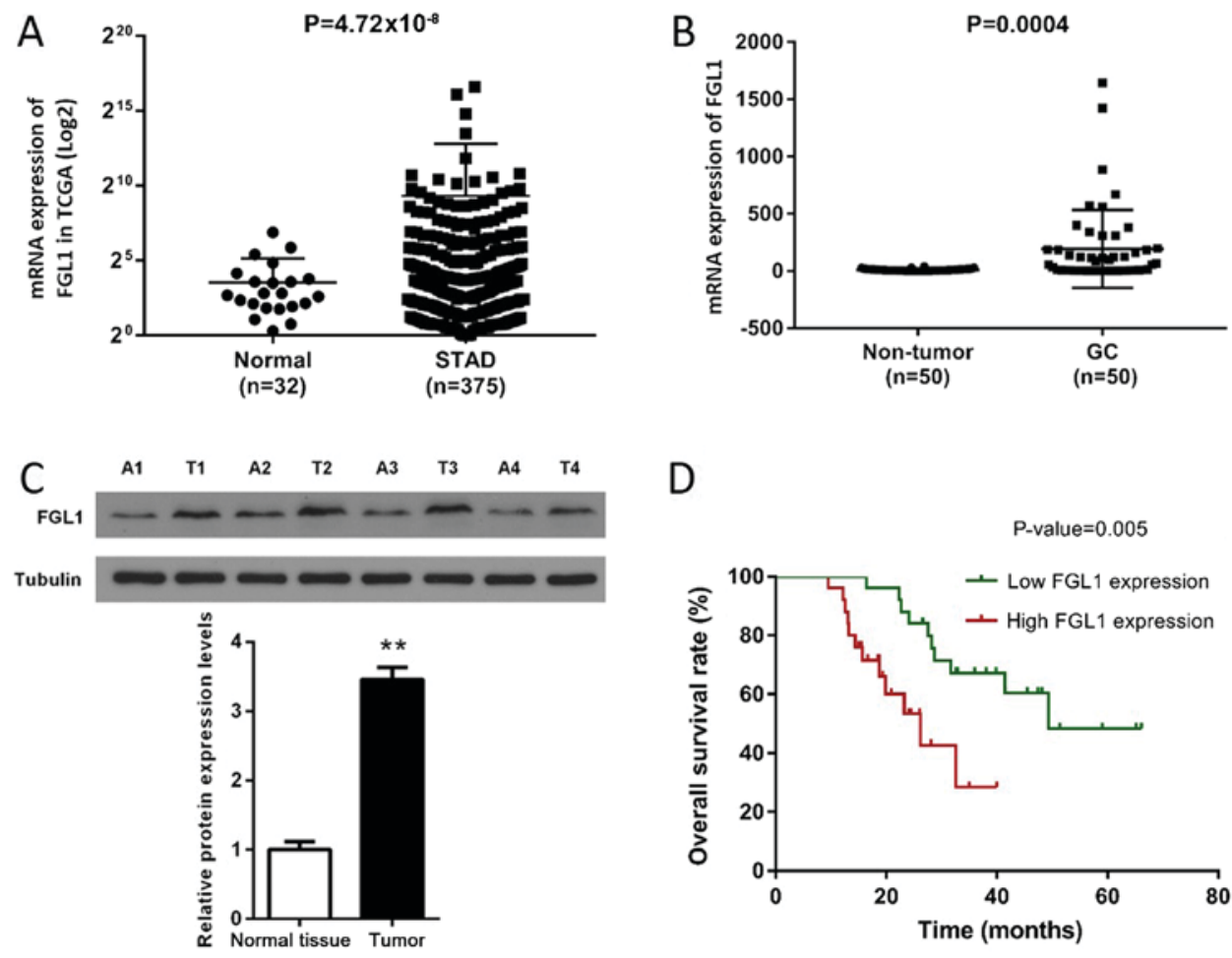

Figure 1. FGL1 is upregulated in gastric cancer tissues and associated with poor prognosis. (A) FGL1 mRNA expression in GC tissues ( $\mathrm{n}=375$ ) compared with normal tissues ( $\mathrm{n}=32$ ) analyzed based on TCGA database. (B) FGL1 mRNA expression in GC tissues ( $\mathrm{n}=50)$ compared with the corresponding non-tumor tissues $(n=50)$ from 50 patients. The expression of FGL1 was determined by reverse transcription-quantitative polymerase chain reaction and normalized to GADPH expression. (C) FGL1 protein expression in GC tissues compared with the corresponding non-tumor tissues. "* $\mathrm{P}<0.01$ vs. normal tissue. (D) High FGL1 expression was correlated with poor prognosis in GC patients. The median was used as the cutoff point of high FGL1 expression (n=25) and low FGL1 expression ( $\mathrm{n}=25)$. STAD, stomach adenocarcinoma; GC, gastric cancer; FGL1, fibrinogen-like-protein 1; TCGA, The Cancer Genome Atlas; A1-A4, adjacent normal tissues 1-4; T1-T4, tumor tissues 1-4.

features was analyzed using Chi-square $\left(\chi^{2}\right)$ test (Table II). The 50 samples were divided into high expression and low expression groups according to the median of FGL1 expression. The results showed that high expression of FGL1 was positive correlated with higher histological grade $(\mathrm{P}=0.041)$, advanced pathologic-stage $(\mathrm{P}=0.023)$ and Lymph node metastasis $(\mathrm{P}=0.021)$. Whereas, no significant association between FGL1 expression and age, sex, pathologic-T, or pathologic-M was observed. These data indicated that the upregulation of FGL1 expression maybe involve in malignant development of GC.

Subsequently, we analyzed the correlation between FGL1 expression and prognosis of the 50 GC patients. The survival curve showed that the overall survival rate (\%) was significantly different between low expression and high expression groups $(\mathrm{P}<0.01)$ and high FGL1 expression was often associated with poor prognosis (Fig. 1D). To investigate whether FGL1 can serve as an independent predictor of gastric adenocarcinoma prognosis, univariate and multivariate Cox proportional hazards analyses was performed on 50 samples (Table III). Univariate analysis found that FGL1 expression, pathologic-M, pathologic- $\mathrm{N}$ and histological grade were obviously related to the overall survival of GC patients $(\mathrm{P}<0.05)$. Multivariate analysis further demonstrated that FGL1 expression, pathologic-M and pathologic-N can serve as independent predictors of poor prognosis in GC patients $(\mathrm{P}<0.05)$. These data suggested that FGL1 possess the potential to serve as a marker for the prognosis of GC patients.
Knockdown of FGL1 suppresses GC cell proliferation. In order to further investigate the expression level of FGL1 in gastric cancer cell lines, qPCR was performed on gastric cancer cell line BGC-823, SGC-7901 and human gastric normal epithelial mucosa cell line GES-1. We found that the expression of FGL1 in BGC-823 and SGC-7901 cells were significantly upregulated compared with that in GES-1 cells $(\mathrm{P}<0.01$, Fig. $2 \mathrm{~A})$. Since the expression of FGL1 in SGC-7901 cells was higher than that in BGC-823, we used SGC-7901 cells to perform subsequent experiments. SGC-7901 cells were transfected with either FGL1 siRNA1 or FGL1 siRNA2, or scramble siRNA. After transfected for $24 \mathrm{~h}$, the expression of FGL1 in SGC-7901 cells transfected with FGL1 siRNA1 decreased significantly compared with the control both at RNA level ( $\mathrm{P}<0.01$, Fig. 2B) and at protein level ( $\mathrm{P}<0.01$, Fig. $4 \mathrm{C}$ and $\mathrm{D})$. While the level of FGL1 was almost not changed in SGC-7901 cells transfected with FGL1 siRNA2 (Fig. 4). Hence cells transfected with FGL1 siRNA1 were used for next experiments.

We explored the effect of FGL1 siRNA 1 on SGC-7901 cell proliferation by CCK8 assay and colony formation assay. We found that the OD value was significantly lower after transfected with FGL1 siRNA1 (siFGL1) for 72 and $96 \mathrm{~h}$ compared with the cells transfected with scramble siRNA (si-con) $(\mathrm{P}<0.01$, Fig. 3A). These data indicated that, siFGL1 played an inhibitory role in SGC-7901 cell proliferation. These results were further confirmed by the colony formation assay. As shown in Fig. 3B and C, knockdown of FGL1 significantly reduced the colony formation rate of SGC-7901 cells $(\mathrm{P}<0.01)$. 
Table III. Univariate and multivariate Cox proportional hazards analyses of clinico-pathological features for the overall survival of 50 GC patients.

\begin{tabular}{|c|c|c|c|c|c|c|}
\hline \multirow[b]{2}{*}{ Variable } & \multicolumn{3}{|c|}{ Univariate analysis } & \multicolumn{3}{|c|}{ Multivariate analysis } \\
\hline & P-value & HR & $95 \% \mathrm{CI}$ & P-value & HR & $95 \% \mathrm{CI}$ \\
\hline FGL1 expression (high/low) & $0.007^{\mathrm{b}}$ & 3.598 & 1.409-9.191 & $0.009^{\mathrm{b}}$ & 4.052 & $1.412-11.629$ \\
\hline Pathologic-T (T1+T2/T3+T4) & 0.646 & 1.250 & $0.482-3.240$ & - & - & - \\
\hline Pathologic-M (M0/M1) & $0.001^{\mathrm{c}}$ & 6.304 & $2.104-18.888$ & $0.030^{\mathrm{a}}$ & 3.592 & $1.135-11.367$ \\
\hline Pathologic-N (N0/N1+N2+N3) & $0.001^{\mathrm{c}}$ & 7.496 & $2.409-23.328$ & $0.022^{\mathrm{a}}$ & 4.403 & $1.237-15.666$ \\
\hline Histological grade $(\mathrm{G} 1+\mathrm{G} 2 / \mathrm{G} 3)$ & $0.009^{\mathrm{b}}$ & 3.748 & $1.400-10.031$ & 0.166 & 2.295 & $0.708-7.437$ \\
\hline Age $(<60 / \geq 60$ years $)$ & 0.512 & 0.751 & $0.319-1.767$ & - & - & - \\
\hline Sex (female/male) & 0.680 & 0.835 & $0.356-1.962$ & - & - & - \\
\hline
\end{tabular}

${ }^{\mathrm{a}} \mathrm{P}<0.05,{ }^{\mathrm{b}} \mathrm{P}<0.01$ and ${ }^{\mathrm{c}} \mathrm{P}<0.001$. GC, gastric cancer; $\mathrm{CI}$, confidence interval; HR, hazard ratio; FGL1, fibrinogen-like-protein 1.

A

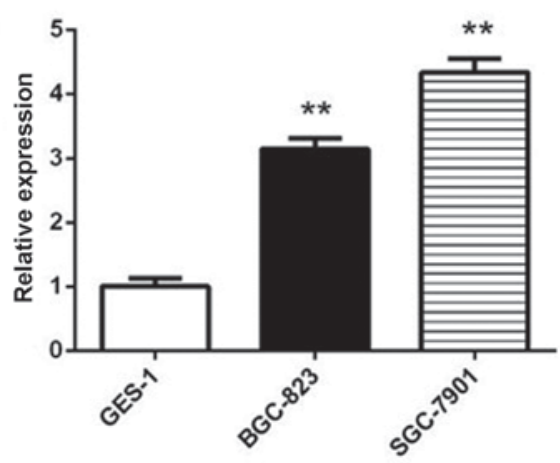

C

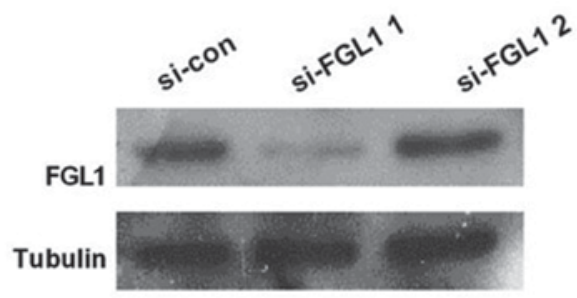

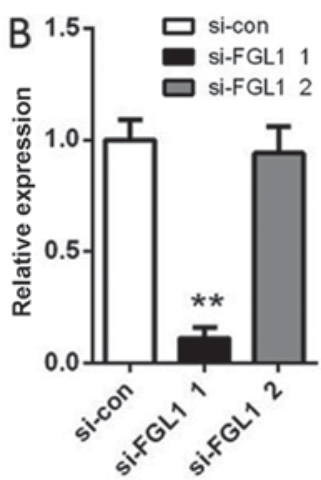

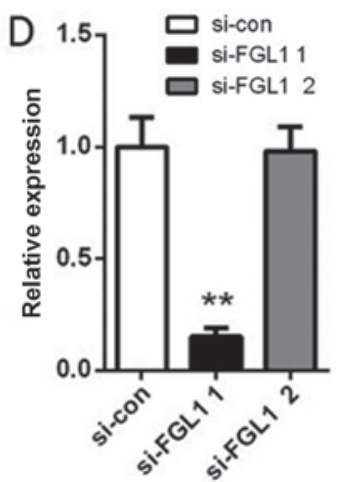

Figure 2. FGL1 siRNA 1 significantly decreases the expression of FGL1 in SGC-7901 cells. (A) The expression levels of FGL1 in the normal gastric mucosal epithelial cell line GES1 and gastric cancer cell lines (BGC-823 and SGC-7901). ${ }^{* *} \mathrm{P}<0.01$ vs. GES-1. (B) The expression level of FGL1 in SGC-7901 cells transfected with FGL1 siRNA analyzed by reverse transcription-quantitative polymerase chain reaction. (C) Western blot analysis of FGL1 expression in SGC-7901 cells transfected with FGL1 siRNA. (D) Quantification of FGL1 protein expression. Data are presented as the mean \pm standard deviation (n=6). ${ }^{* *} \mathrm{P}<0.01$ vs. si-con. si-/siRNA, small inferring RNA; si-con, scramble siRNA control; FGL1, fibrinogen-like-protein 1; si-FGL1 1/2, FGL1 siRNA 1/2.

Knockdown of FGL1 suppressed SGC-7901 cell migration and invasion. To assess the effect of FGL1 on SGC-7901 cell migration, scratch wound healing assay and Transwell migration assay were utilized. Compared with the control group, a narrower migratory distance was observed after silencing of FGL1 ( $\mathrm{P}<0.01$, Fig. 4A and B). We also identified that knockdown of FGL1 obviously reduced the number of migrated SGC-7901 cells ( $\mathrm{P}<0.01$, Fig. 4C and D). These data indicated that FGL1 play a promoting role in SGC-7901 cell migration.

The invasion ability of SGC-7901 cells was tested using Transwell invasion assay. As shown in Fig. 4C and D, the number of invaded cells in siFGL1 group declined remarkably compared with the si-con group $(\mathrm{P}<0.01)$. These results demonstrated that FGL1 also have a promoting effect on SGC-7901 cell invasion.

FGL1 involved in the regulation of epithelial to mesenchymal transitions in SGC-7901 cells. Epithelial to mesenchymal transitions (EMT) is a process that epithelial cells transform into mesenchymal cells under physiological or pathological conditions, which allows for cell movement. We further explored the effects of FGL1 on EMT of SGC-7901 cells. 

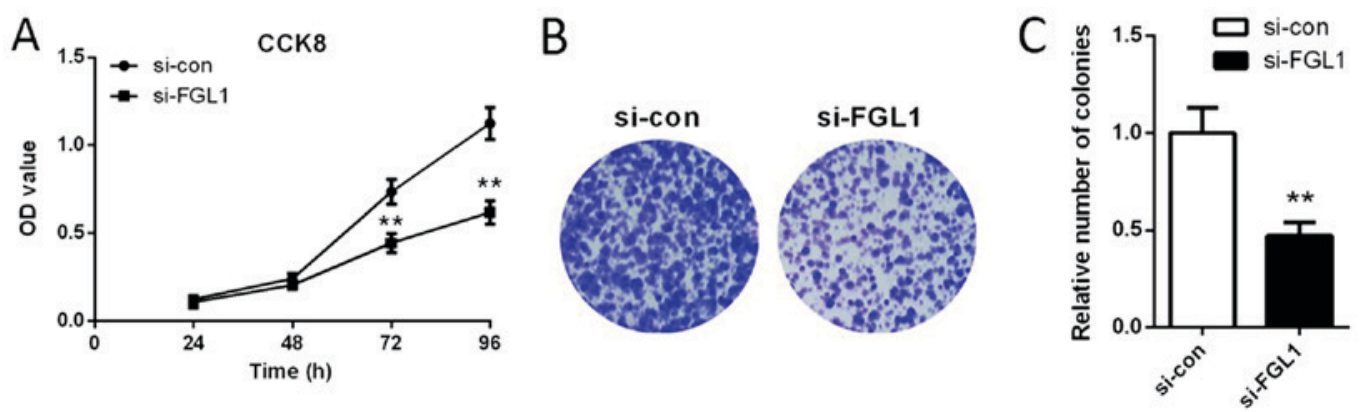

Figure 3. FGL1 siRNA 1 inhibits SGC-7901 cell proliferation. (A) The effect of FGL1 siRNA 1 on SGC-7901 cell proliferation tested by Cell Counting Kit-8 assay. (B) Colony-forming assay was performed to determine the effect of FGL1 siRNA 1 on SGC-7901 cell proliferation (magnification, x2). (C) Quantification of the colonies stained by crystal violet. Data are presented as the mean \pm standard deviation $(\mathrm{n}=6) .{ }^{* * *} \mathrm{P}<0.01$ vs. si-con. si-/siRNA, small inferring RNA; si-con, scramble siRNA control; FGL1, fibrinogen-like-protein 1; si-FGL1, FGL1 siRNA 1; OD, optical density.

A

$\mathrm{Oh}$

$24 \mathrm{~h}$

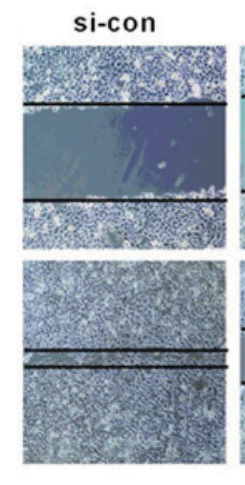

C

Invasion

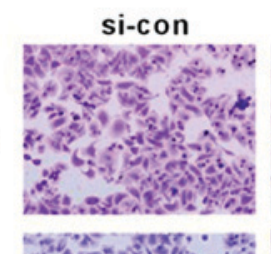

si-FGL1

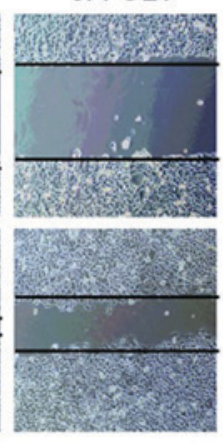

si-FGL1

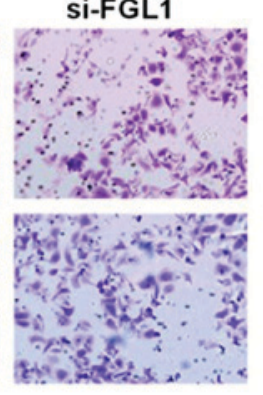

B

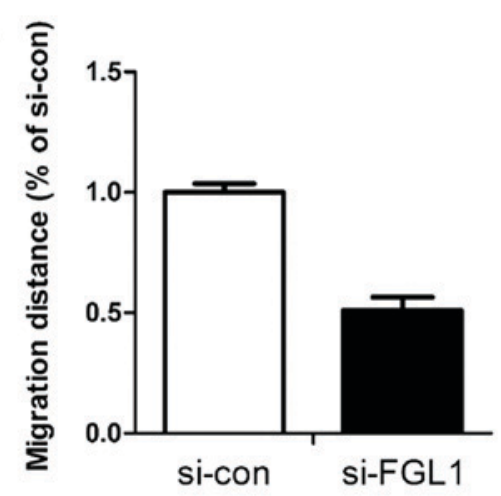

D

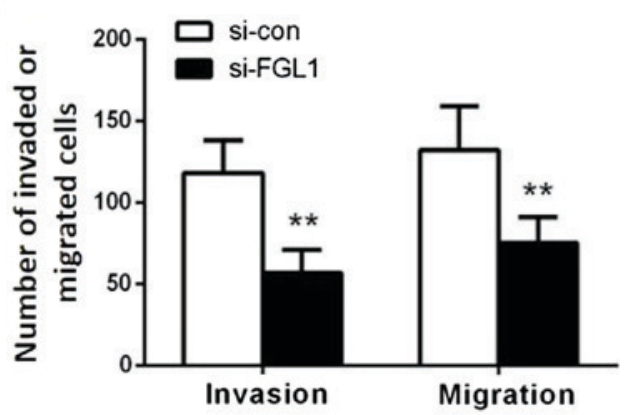

Figure 4. FGL1 siRNA 1 inhibits SGC-7901 cell migration and invasion. (A) SGC-7901 cell migration was assessed following silencing of FGL1 by wound healing assays at 0 and $24 \mathrm{~h}$ post-scratching (magnification, x100). (B) Relative migration distance of SGC-7901 cells. (C) Transwell invasion and migration assays were performed to determine SGC-7901 cell invasion and migration following transfection with FGL1 siRNA 1 for $24 \mathrm{~h}$ (magnification, x200). (D) Quantification of invaded and migrated cells. Data are presented as the mean \pm standard deviation $(\mathrm{n}=5) .{ }^{* *} \mathrm{P}<0.01 \mathrm{vs}$. si-con. si-con. si-/siRNA, small inferring RNA; si-con, scramble siRNA control; FGL1, fibrinogen-like-protein 1; si-FGL1, FGL1 siRNA 1.

Morphological observation found that cells in si-con group include spindle-shaped cells, while cells with depleting FGL1 were pebble-alike (Fig. 5A). Western blot analysis showed that knock down of FGL1 lead to an obviously increase in E-cadherin expression $(\mathrm{P}<0.01)$ and a significant decrease in $\mathrm{N}$-cadherin $(\mathrm{P}<0.01)$ and Vimentin expression $(\mathrm{P}<0.01)$ compared with the si-con group (Fig. 5B and C). These results implied that FGL1 was indeed involved in the regulation of EMT in SGC-7901 cells.

\section{Discussion}

This study aimed to elucidate the role of FGL1 in GC. We identified that FGL1 was upregulated in GC tissues and high FGL1 expression was significantly correlated with poor prognosis. Moreover, an inhibitory effect on SGC-7901 cell proliferation, invasion and migration was observed after silencing FGL1.

Previous studies of FGL1, have mainly focused on its role in stimulating tritiated thymidine uptake into hepatocytes, rescuing animals from liver failure $(9,10,12)$. Demchev et al (8) have reported that FGL1 was also expressed in brown adipose tissue and the expression was enhanced following liver injury, suggesting a correlation between the injured liver and adipose tissues. Further experiments indicated that FGL1 plays a role in metabolism and liver regeneration (8). At the present study, we reported the upregulation of FGL1 in GC at the first time, indicating FGL1 may be a promotor of GC. While, it has been reported that the level of FGL1 in hepatocellular carcinoma 
A

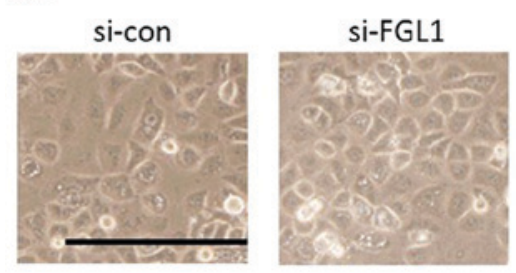

B

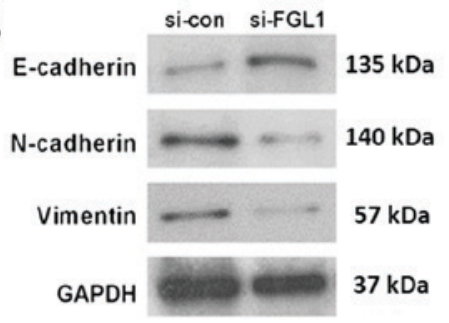

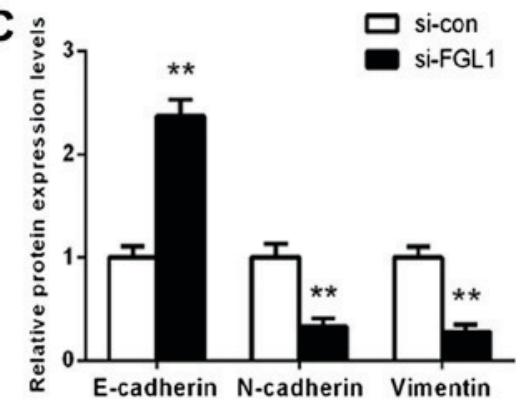

Figure 5. Silencing FGL1 inhibits EMT in SGC-7901 cells. (A) Morphologies of SGC-7901 cells treated with scramble siRNA control and FGL1 siRNA 1. Scale bar $=200 \mu \mathrm{m}$. (B) The expression of the EMT-associated proteins, E-cadherin, N-cadherin and Vimentin were detected in SGC-7901 cells transfected with FGL1 siRNA 1 and scramble siRNA by western blotting. (C) Quantification of E-cadherin, N-cadherin and Vimentin protein expression. Data are presented as the mean \pm standard deviation $(\mathrm{n}=6) .{ }^{* *} \mathrm{P}<0.01$ vs. si-con. si-con. si-/siRNA, small inferring RNA; si-con, scramble siRNA control; FGL1, fibrinogen-like-protein 1; si-FGL1, FGL1 siRNA 1; EMT, epithelial-mesenchymal transitions.

(HCC) was downregulated and it may serves as a tumor inhibitor in HCC through an Akt dependent mechanism $(13,14)$. These difference indicating that FGL1 may be either upregulated or downregulated depending on the cancer type.

To further explore the clinical value of FGL1, the relationship between FGL1 expression and the clinical and pathological factors of GC was analyzed. Our results suggested that FGL1 was obviously correlated with histological grade, pathologic-stage and Lymph node metastasis. However, no significant associations between FGL1 the following factors, including age, sex, pathologic-T, or pathologic-M were found. Simultaneously, we identified that high expression of FGL1, pathologic-M and pathologic-N can serve as independent prognostic risk factors in GC.

In order to investigate the biological effect of FGL1 in GC cell lines, we performed siRNA knockdown of FGL1 in SGC-7901 cells. By colony formation assay and CCK8 assay, we found that silencing FGL1 significantly suppressed SGC-7901 cell proliferation $(\mathrm{P}<0.01)$. Furthermore, the results of wound healing assay and Transwell invasion and migration assay indicated that knockdown of FGL1 obviously decreased SGC-7901 cell invasion and migration $(\mathrm{P}<0.01)$ in vitro. These observations indicated that FGL1 was probably an oncogene which play a promoting role in GC cell proliferation, invasion and migration. Furthermore, these results confirmed that high expression of FGL1 was correlated with poor prognosis in GC patients.

EMT has been widely recognized as an indispensable member in tumor invasion and metastasis $(15,16)$. E-cadherin, $\mathrm{N}$-cadherin and Vimentin are important markers of EMT (17-19). Loss of E-cadherin, an important feature of EMT, has been identified to relate to invasive and undifferentiated phenotype in many types of tumors (19-21). Upregulated expression of $\mathrm{N}$-cadherin and Vimentin was also a key characterization of EMT $(22,23)$. In the present study, we found that the expression of E-cadherin was significantly upregulated and the levels of N-cadherin and Vimentin were downregulated obviously in SGC-7901 cells after knocking down of FGL1. This result indicated that FGL1 played a promoting role in tumor invasion and metastasis and further confirmed the results we obtained in cell migration and invasion assays. Moreover, this is the first time to demonstrate that FGL1 could regulate EMT.

In summary, our results demonstrated that the expression of FGL1 was upregulated in GC tissues as well as GC cell lines, and high expression of FGL1 can serve as an independent predictor of poor prognosis for GC patients. Silencing FGL1 lead to an inhibitory effect on GC cell proliferation, migration and invasion. Our results suggested that FGL1 has the potential to be a predictor of prognosis in GC patients as well as a target for the treatment of GC.

\section{Acknowledgements}

The authors would like to thank Zhengzhou Central Hospital Affiliated to Zhengzhou University (Henan, China) for providing the experimental platform.

\section{Funding}

No funding was received.

\section{Availability of data and materials}

All data generated or analyzed during this study are included in this published article.

\section{Authors' contributions}

YZ and JHC contributed to the conception and design of the present study. YZ and HXQ conducted the experiments, and analyzed and interpreted the data. YTZ and LH assisted with data collection and bioinformatics analysis. YZ was responsible for drafting the manuscript. HXQ, YTZ and LH revised the manuscript. JHC critically revised the manuscript for important intellectual content and gave final approval of the version to be published.

\section{Ethics approval and consent to participate}

The present study was approved by the Ethics Committee of Zhengzhou Central Hospital Affiliated to Zhengzhou University (Henan, China). Written informed consent was obtained from all patients.

\section{Consent for publication}

Not applicable. 


\section{Competing interests}

The authors declare that they have no competing interests.

\section{References}

1. Sjoquist KM and Zalcberg JR: Gastric cancer: Past progress and present challenges. Gastric Cancer 18: 205-209, 2015.

2. Torre LA, Bray F, Siegel RL, Ferlay J, Lortet-Tieulent J and Jemal A: Global cancer statistics, 2012. CA Cancer J Clin 65 87-108, 2015.

3. Siegel R, Ma J, Zou Z and Jemal A: Cancer statistics, 2014. CA Cancer J Clin 64: 9-29, 2014.

4. Lin MT, Lin BR, Chang CC, Chu CY, Su HJ, Chen ST, Jeng YM and Kuo ML: IL-6 induces AGS gastric cancer cell invasion via activation of the c-Src/RhoA/ROCK signaling pathway. Int J Cancer 120: 2600-2608, 2007.

5. Dassen AE, Lemmens VE, van de Poll-Franse LV, Creemers GJ, Brenninkmeijer SJ, Lips DJ, Vd Wurff AA, Bosscha K and Coebergh JW: Trends in incidence, treatment and survival of gastric adenocarcinoma between 1990 and 2007: A population-based study in The Netherlands. Eur J Cancer 46: 1101-1110, 2010.

6. Szász AM, Lánczky A, Nagy Á, Förster S, Hark K, Green JE, Boussioutas A, Busuttil R, Szabó A and Győrffy B: Cross-validation of survival associated biomarkers in gastric cancer using transcriptomic data of 1,065 patients. Oncotarget 7 : 49322-49333, 2016.

7. Yamamoto T, Gotoh M, Sasaki H, Terada M, Kitajima M and Hirohashi S: Molecular cloning and initial characterization of a novel fibrinogen-related gene, HFREP-1. Biochem Biophys Res Commun 193: 681-687, 1993.

8. Demchev V, Malana G, Vangala D, Stoll J, Desai A, Kang HW, Li Y, Nayeb-Hashemi H, Niepel M, Cohen DE and Ukomadu C: Targeted deletion of fibrinogen like protein 1 reveals a novel role in energy substrate utilization. PLoS One 8: e58084, 2013.

9. Hara H, Yoshimura H, Uchida S, Toyoda Y, Aoki M, Sakai Y, Morimoto S and Shiokawa K: Molecular cloning and functional expression analysis of a cDNA for human hepassocin, a liver-specific protein with hepatocyte mitogenic activity. Biochim Biophys Acta 1520: 45-53, 2001.

10. Li CY, Cao CZ, Xu WX, Cao MM, Yang F, Dong L, Yu M, Zhan YQ, Gao YB, Li W, et al: Recombinant human hepassocin stimulates proliferation of hepatocytes in vivo and improves survival in rats with fulminant hepatic failure. Gut 59: 817-826, 2010.
11. Livak KJ and Schmittgen TD: Analysis of relative gene expression data using real-time quantitative PCR and the 2(-Delta Delta C(T)) method. Methods 25: 402-408, 2001.

12. Liu Z and Ukomadu C: Fibrinogen-like protein 1, a hepatocyte derived protein is an acute phase reactant. Biochem Biophys Res Commun 365: 729-734, 2008

13. Nayeb-Hashemi H, Desai A, Demchev V, Bronson RT, Hornick JL, Cohen DE and Ukomadu C: Targeted disruption of fibrinogen like protein-1 accelerates hepatocellular carcinoma development. Biochem Biophys Res Commun 465: 167-173, 2015.

14. Yan J, Yu Y, Wang N, Chang Y, Ying H, Liu W, He J, Li S, Jiang W, Li Y, et al: LFIRE-1/HFREP-1, a liver-specific gene, is frequently downregulated and has growth suppressor activity in hepatocellular carcinoma. Oncogene 23: 1939-1949, 2004.

15. Grunert S, Jechlinger $M$ and Beug H: Diverse cellular and molecular mechanisms contribute to epithelial plasticity and metastasis. Nat Rev Mol Cell Biol 4: 657-665, 2003.

16. Giannelli G, Koudelkova P, Dituri F and Mikulits W: Role of epithelial to mesenchymal transition in hepatocellular carcinoma. J Hepatol 65: 798-808, 2016.

17. Prasad CP, Rath G, Mathur S, Bhatnagar D, Parshad R and Ralhan R: Expression analysis of E-cadherin, Slug and GSK3beta in invasive ductal carcinoma of breast. BMC Cancer 9: 325, 2009.

18. Zhang W, Altholland A, Margulis A, Shamis Y, Fusenig NE, Rodeck U and Garlick JA: E-cadherin loss promotes the initiation of squamous cell carcinoma invasion through modulation of integrin-mediated adhesion. J Cell Sci 119: 283-291, 2006.

19. Nakajima S, Doi R, Toyoda E, Tsuji S, Wada M, Koizumi M, Tulachan SS, Ito D, Kami K, Mori T, et al: N-cadherin expression and epithelial-mesenchymal transition in pancreatic carcinoma. Clin Cancer Res 10: 4125-4133, 2004.

20. Zhou J, Tao D, Xu Q, Gao Z and Tang D: Expression of E-cadherin and vimentin in oral squamous cell carcinoma. Int J Clin Exp Pathol 8: 3150-3154, 2015.

21. Xie X, Zheng X, Wang J and Chen L: Clinical significance of Twist, E-cadherin, and $\mathrm{N}$-cadherin protein expression in endometrioid adenocarcinoma. J Cancer Res Ther 13: 817-822, 2017.

22. Avila D: N-cadherin and vimentin expression in small rounded-shaped cells of non-functioning human pituitary adenomas. Int J Clin Exp 9: 7854-7866, 2016.

23. Satelli A and Li S: Vimentin in cancer and its potential as a molecular target for cancer therapy. Cell Mol Life Sci 68: 3033-3046, 2011.

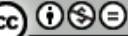

This work is licensed under a Creative Commons Attribution-NonCommercial-NoDerivatives 4.0 International (CC BY-NC-ND 4.0) License. 